\title{
Digitales Storytelling: Programmieren lernen durch Geschichtenerzählen
}

\author{
Mario Eich, Julia Thomas, Christian Fischer \\ University of Tübingen
}

Abstract: Vor 44.000 Jahren begannen Menschen sich Geschichten zu erzählen und damit Wissen und Werte an nachfolgende Generationen weiterzugeben. Aus Höhlenwänden wurde im Laufe der Zeit Papier und nun mit Computern begeistern sich viele Schülerinnen und Schüler für das „Digital Storytelling“. Plattformen wie Scratch verbinden das Storytelling, wie das Geschichtenerzählen auch genannt wird, mit multimedialer Technik - und vermitteln so ganz nebenbei auch spielerisch Kenntnisse im Programmieren.

\section{Klassisches Storytelling mit Stift und Papier}

„Schreibe eine kurze Geschichte über etwas, das du am vergangenen Wochenende erlebt hast", lautet eine klassische Aufgabenstellung an Schülerinnen und Schüler. Besonders in Grundschulen wird das Geschichtenerzählen genutzt, um Kinder an das Schreiben von Texten heranzuführen und sie zu motivieren, eigene Erlebnisse festzuhalten. Um die Kreativität anzuregen, wird das Geschichtenerzählen zudem häufig mit dem Zeichnen von Bildern verknüpft. Das Schreiben und Zeichnen von Geschichten regt nicht nur dazu an, Erlebtes zu reflektieren, sondern Ereignisse auch in eigenen Worten wiederzugeben und auf die wichtigsten Bestandteile zu reduzieren. Mit der Aufgabe, ein Ereignis als Geschichte aufzuschreiben, setzt daher ein aktiver (Re-)Konstruktionsprozess ein. Während das Geschichtenerzählen im Unterricht klassischerweise noch auf Papier stattfindet, heben digitale Lerntools wie Scratch Geschichten auf eine neue, multimediale Ebene. 


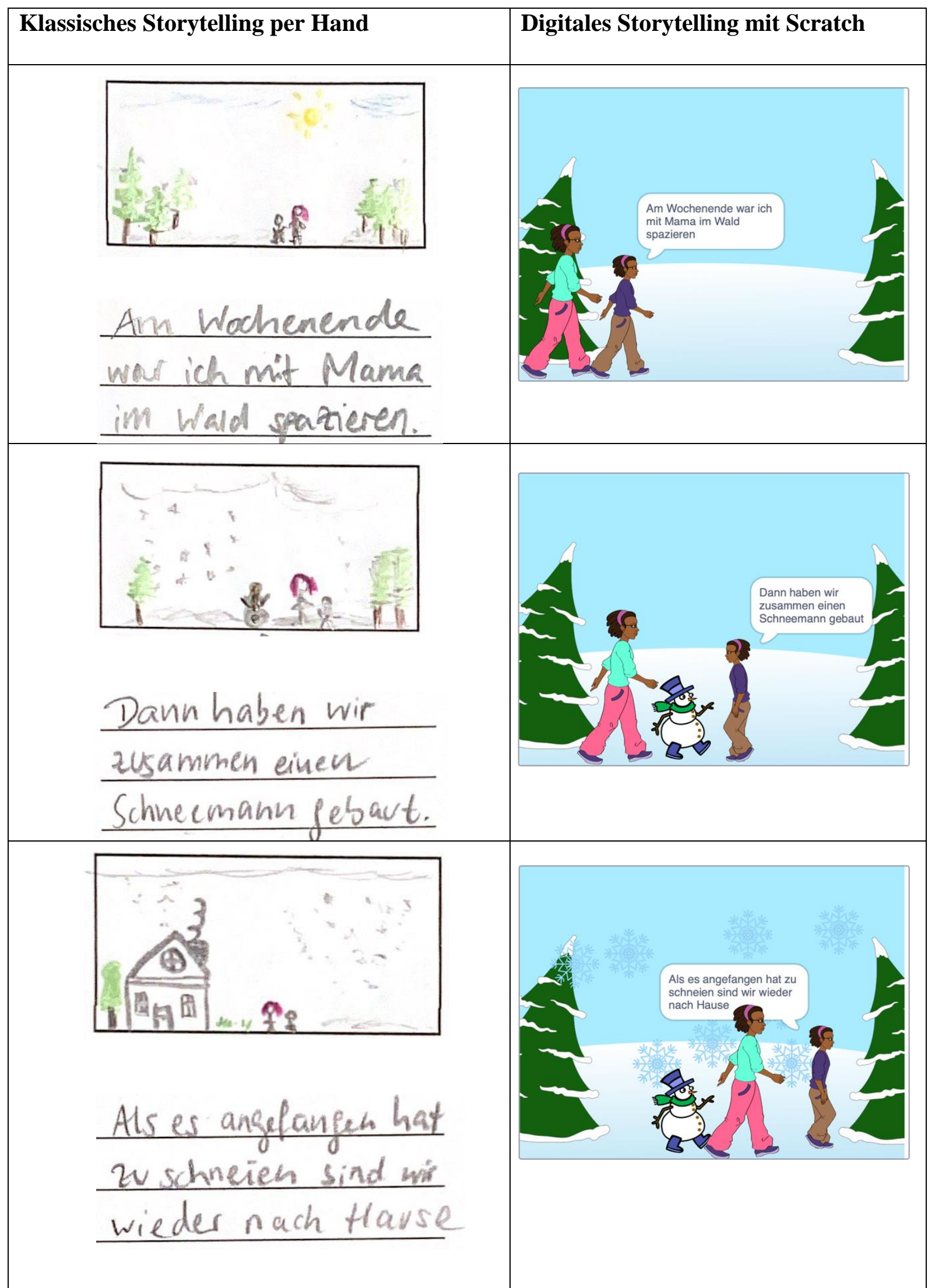

Abbildung 1: Gegenüberstellung vom analogen und digitalen Storytelling. 


\section{Multimediale Geschichten mit Video und Ton}

Beim digitalen Storytelling geht es nicht nur darum, eigene Erlebnisse in schönen

Geschichten zu erzählen und die Kreativität der Lernenden zu fördern, sondern auch um ein besseres Verständnis von Erzählstrukturen und das Einbinden von multimedialen Elementen (z.B. Robin, 2008; Sadik, 2008). Lernende müssen das Erlebte in kurze Abschnitte teilen, die in Lern-Tools wie Scratch in Programmiersprache übersetzt und dargestellt werden können. Um ihre Erlebnisse anschaulich wiederzugeben, müssen sie diese daher zunächst verstehen und in eine chronologische Reihenfolge bringen. Durch die Gliederung in kleine Einzelelemente und das Zusammenbauen dieser Elemente entsteht schließlich eine multimediale Geschichte voller Details. Dabei wird neben der Kreativität auch die Neugier der Lernenden auf das

Programmieren geweckt. Lern-Tools wie Scratch verbinden traditionelle Komponenten der Erzählung mit spielerischen Lernangeboten aus dem Bereich Informatik und führen die Schülerinnen und Schüler so an gesellschaftlich immer wichtiger werdende digitale Kompetenzen heran.
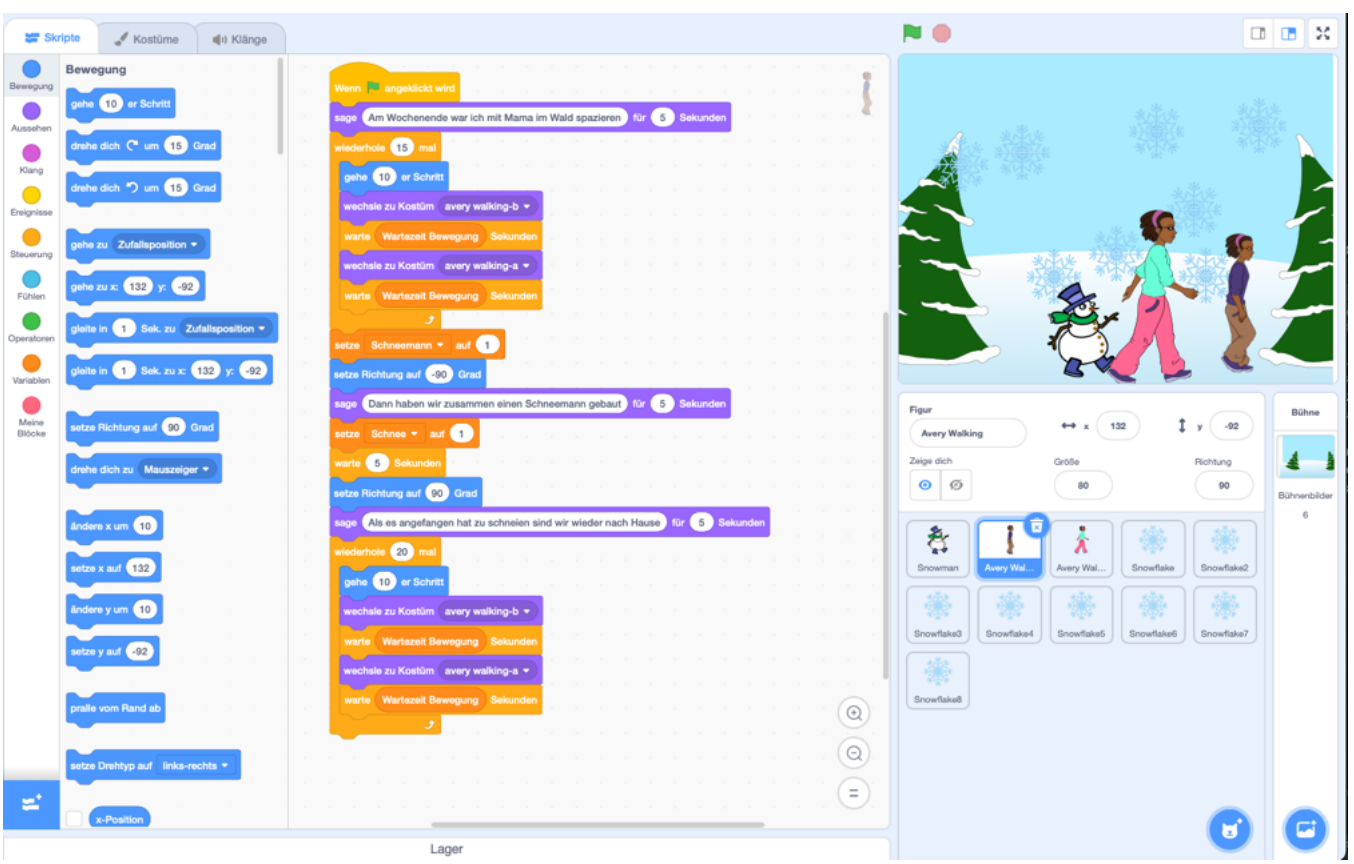

Abbildung 2: Programmieroberfläche in Scratch. 


\section{Infobox: Was ist Scratch?}

Scratch ist eine lernorientierte grafische Programmiersprache für Kinder und Jugendliche, die 2007 von der Media Lab Lifelong Kindergarden Group am MIT veröffentlicht wurde und seitdem stetig erweitert und verbessert wird. Mit drei pädagogischen Kerngedanken „Low Floors“, „Wide Walls“ und „High Ceiling“ - verfolgt Scratch das Ziel, Kinder und Jugendliche zum spielerischen Lernen zu ermutigen (Resnick et al., 2009). „Low Floor“ bedeutet, dass der Einstieg in die Programmiersprache keine Vorkenntnisse erfordert und es entsprechend leicht fällt, bereits zu Beginn kleinere Projekte mit schnellen Erfolgserlebnissen zu realisieren (Karossa, Ferdinand, \& Fischer, 2020). Unter dem Begriff „Wide Walls“ bietet Scratch eine vielfältige Bandbreite an Möglichkeiten, um Projekte und Aufgabenstellungen zu realisieren. So können Figuren und Gegenstände nicht nur zu einer digitalen Story verknüpft werden, sondern beispielsweise auch ein „Jump and Run Spiel“ programmiert werden. Damit kann Scratch unabhängig von Interessen und der Persönlichkeit der Kinder und Jugendlichen eingesetzt werden. Der Komplexität der Projekte sind zudem kaum Grenzen gesetzt, wodurch immer wieder neue Anreize geschaffen werden, verschiedene Programmierkonzepte der Informatik spielerisch zu erlernen und anzuwenden (,High Ceiling“). Scratch ist einfach, multimedial, kostenlos und mehrsprachig.

\section{Besondere Dynamik durch Bewegung}

Mit Lerntools wie Scratch können Lernende ihren Geschichten eine Dynamik verleihen, die durch das analoge Zeichnen von Bildern kaum umsetzbar ist. So können beispielsweise Bewegungen, Formen, Hintergründe, Stimmen und Geräusche in die Geschichte integriert werden. Viele Elemente können spielerisch verändert und hinzugefügt werden, um die 
Kreativität der Lernenden zu fördern und ihre Ideen aufzugreifen. Gleichzeitig lernen die

Schülerinnen und Schüler spielerisch wichtige Elemente des Programmierens kennen.

Zudem ist die Arbeit mit Scratch gleichzeitig reaktiv und interaktiv. Reaktiv, weil die

Lernenden auf die gegebenen Möglichkeiten in Scratch und die Schnittstellen des Programms reagieren sowie mögliche Darstellungsschwierigkeiten überwinden müssen. Interaktiv, weil nicht das Aufschreiben der eigenen Erlebnisse im Vordergrund steht, sondern das Verständnis für Erzählbausteine, die sich anschließend in eine Programmiersprache übersetzen lassen, um eine Geschichte möglichst anschaulich darstellen zu können. Durch die Community der Plattform bietet Scratch den Schülerinnen und Schülern zudem die Möglichkeit, ihre erstellten Storys sowohl mit der eigenen Klasse, als auch mit Freunden oder ihrer Familie zu teilen.

\section{Vorteile von Scratch im Unterricht nutzen}

In den Bildungsplänen ist das Verfassen von Erlebniserzählungen bis zur 6. Jahrgangsstufe verankert (Zentrum für Schulqualität und Lehrerbildung, 2021). Da auf Scratch Accounts für Lehrkräfte angelegt werden können, ist es möglich, die Projekte der Schülerinnen und Schüler koordiniert zu steuern und zu verwalten, weshalb sich Scratch ideal für den Einsatz im Klassenverband eignet. Klassisches und digitales Storytelling schließen sich dabei jedoch nicht aus: Sie können Hand in Hand gehen und sich je nach Gegebenheit ergänzen. Scratch kann folglich in den Unterricht integriert werden, den Unterricht ergänzen oder eine Alternative zum klassischen Geschichtenerzählen darstellen. Empirische Studien zeigen, dass die Verwendung von Scratch die Kreativität von Kindern und Jugendlichen erhöht (z.B. Maloney et al., 2008). Je länger das Tool genutzt wird, desto häufiger werden auch komplexere Funktionen des Programmierens wie Schleifen oder Variablen erlernt. Scratch fördert damit nicht nur die Kreativität und Experimentierfreudigkeit der Lernenden, sondern auch ihr Kompetenzerleben. 


\section{References}

Karossa, N., Ferdinand, J., \& Fischer, C. (2020). Programmieren kinderleicht: Schnelle Erfolgserlebnisse mit Scratch. OSF Preprints. https://doi.org/10.31219/osf.io/zs2a8

Maloney, J. H., Peppler, K., Kafai, Y., Resnick, M., \& Rusk, N. (2008, March). Programming by choice: urban youth learning programming with scratch. In Proceedings of the 39th SIGCSE Technical Symposium on Computer Science Education, 367-371.

Resnick, M., Maloney, J., Monroy-Hernández, A., Rusk, N., Eastmond, E., Brennan, K., ... \& Kafai, Y. (2009). Scratch: programming for all. Communications of the ACM, 52(11), 6067.

Robin, B. R. (2008). Digital storytelling: A powerful technology tool for the 21 st century classroom. Theory into Practice, 47(3), 220-228.

Sadik, A. (2008). Digital storytelling: A meaningful technology-integrated approach for engaged student learning. Educational Technology Research and Development, 56(4), 487-506

Zentrum für Schulqualität und Lehrerbildung (2021). Bildungsplan 2016, Retrieved from: http://www.bildungsplaene-bw.de (abgerufen am 02.03.2021) 